\title{
Graphite-based Lithium-free 3D Hybrid Anodes for High Energy Density All-Solid-State Batteries
}

Xing Xing, Yejing Li, Shen Wang, Haodong Liu, Zhaohui Wu, Sicen Yu, John Holoubek, Hongyao Zhou, Ping Liu*

Department of Nanoengineering, University of California, San Diego, La Jolla, CA 92093

\section{SUPPORTING INFORMATION}

Experimental section

Materials and synthesis. All chemicals were purchased from Sigma-Aldrich unless specified and used without purification. All the cell fabrications and testing were performed inside an Argonfilled glove box (MBRAUN, MB200B, $\mathrm{H}_{2} \mathrm{O}<0.5 \mathrm{ppm}, \mathrm{O}_{2}<0.5 \mathrm{ppm}$ ). High purity LPS powder was purchased from the NEI corporation (USA) and used as-is.

Anode composite: Graphite/LPS anode composite were prepared with graphite and LPS powder in a ratio of 1:1 by weight. The mixture was hand ground with an agate mortar for 20 minutes to make a homogeneous composite.

Cathode composite: A solution method was employed to coat $\mathrm{LiNbO}_{3}$ on $\mathrm{NCA}$ particles. First, lithium ethoxide and niobium ethoxide were dissolved in anhydrous ethanol. NCA powder was then added into the solution and stirred for one hour. The dried powder was collected by evaporating the ethanol using a rotary evaporator followed by heat-treatment of the powder at 450 
${ }^{\circ} \mathrm{C}$ for one hour, yielding $\mathrm{LiNbO}_{3}$ coated NCA. The coated powder was dried overnight at $100{ }^{\circ} \mathrm{C}$ under vacuum before transferring to the inside of the glove box for storage and fabrication of solidstate batteries. Then, NCA cathode composite was prepared with coated NCA, LPS, vapor growth carbon fiber (VGCF) in a ratio of 70:28:2. All components were hand ground to make a homogeneous mixture.

Characterizations and electrochemical analysis. The morphologies of the cycled anode were characterized using scanning electron microscopy (FEI Quanta 250 SEM) coupled with an energy dispersive X-ray spectrometer (EDX) to determine the chemical composition of the samples. The samples were tested under $10 \mathrm{kV}$ and $0.1 \mathrm{nA}$ conditions. The cross-section images were collected using focused ion beam-scanning electron microscopy (FIB-SEM, FEI Scios, Scios DualBeam operating at $5 \mathrm{kV}$ and $0.1 \mathrm{nA}$ conditions). The cross-sections were first cut under $30 \mathrm{nA}$, followed by a $0.1 \mathrm{nA}$ surface cleaning process. All the samples were prepared inside an Ar environment glove box and sealed inside a bag made of aluminum laminate sheets. During transfer, the samples was briefly exposed to air.

The electrolyte pellets were prepared using a $13 \mathrm{~mm}$ custom-made polyether ether ketone (PEEK) die mold and titanium (Ti) plungers. LPS electrolyte powder (150mg) was pressed at $400 \mathrm{MPa}$ for 3 minutes with a hydraulic press inside the mold to obtain a $\sim 800 \mu \mathrm{m}$ thick electrolyte pellet. Then, a certain amount of cathode and anode materials were filled on both sides of the pellet and pressed at $400 \mathrm{MPa}$ to obtain a three-layered pellet. Two Ti plungers were used as current collectors while the full cell was measured under a $90 \mathrm{MPa}$ stacking pressure. This pressure was found to offer optimum cycling performance when a 3D anode is used. We note the value to be considerably 
higher than those desired for a Li metal anode. In this case, the graphite is expected to carry most of the mechanical load.

All the galvanostatic charge/discharge tests were conducted inside a $35^{\circ} \mathrm{C}$ oven, using LAND battery testers (Wuhan, China). The critical current density tests of Li-LPS-Li cell and 3D-LPS$3 \mathrm{D}$ cell were conducted with a $0.1 \mathrm{~mA} \mathrm{~cm}^{-2}$ increment each cycle until the cell shorted. The time for each charge and discharge process was 1 hour. For Li-Cu and Li-3D half-cell tests, the cell areal discharge capacity was fixed with a certain value, but the cell charge process was controlled by a voltage cut-off $(1 \mathrm{~V})$ to estimate the Coulombic efficiency of Li plating and stripping. The NCA-Cu and NCA-3D cells were tested in a voltage range of $2.5-4.3 \mathrm{~V}$ vs. $\mathrm{Li} / \mathrm{Li}^{+}$at room temperature with a current density of $0.1 \mathrm{~mA} \mathrm{~cm}^{-2}$. While $\mathrm{Cu}$ might react with sulfide under extended storage, such reactivity is not significant when $\mathrm{Cu}$ is biased at near Li metal potential. This is confirmed by a comparison with a Ti electrode as detailed in Discussion Six.

Electrochemical impedance was measured using electrochemical impedance spectroscopy (EIS, BioLogic, VMP300t) in a frequency range from $7 \mathrm{MHz}$ to $100 \mathrm{mHz}$. 


\section{Discussion one:}

To demonstrate the necessity of LPS SSE as the ionic conductor in the anode network, a pure graphite anode without LPS was tested in a half cell with lithium metal counter electrode. The voltage profile in Figure S1a shows that after the full lithiation of graphite an additional $1 \mathrm{mAh}$ $\mathrm{cm}^{-2}$ of Li metal was deposited. To examine the Li metal distribution, the cell was disassembled to characterize its morphology/composition under scanning electron microscopy (SEM). Figure S1c displays an area where the electrolyte layer is partially removed to unveil the underlying graphite anode. Energy-dispersive X-ray spectroscopy (EDX) mapping (Figure S1d) demonstrates that island-like features represented by the dashed white lines are rich in oxygen without other elements (C, P, and S). The high concentration of oxygen is usually correlated with lithium metal (oxidized due to air exposure during sample transfer) ${ }^{1}$. It is evident that Li metal was deposited at the interface between graphite and SSE. To examine whether lithium also deposits in the bulk of the graphite anode, the anode surface was gently scratched to unveil the underlying layer (Figure $\mathrm{S} 1 b)$. While the top surface has a golden color, which is attributed to $\mathrm{LiC}_{6}{ }^{2-6}$, the underlayer appears to be black, indicative of poor lithiation graphite due to a lack of ion conductivity (all lithium ions have to be transported through graphite itself). We thus conclude that the pure lithiated graphite does not have sufficient ionic conductivity to allow complete lithiation of the graphite layer and guide lithium metal deposition away from the anode/electrolyte interface. 

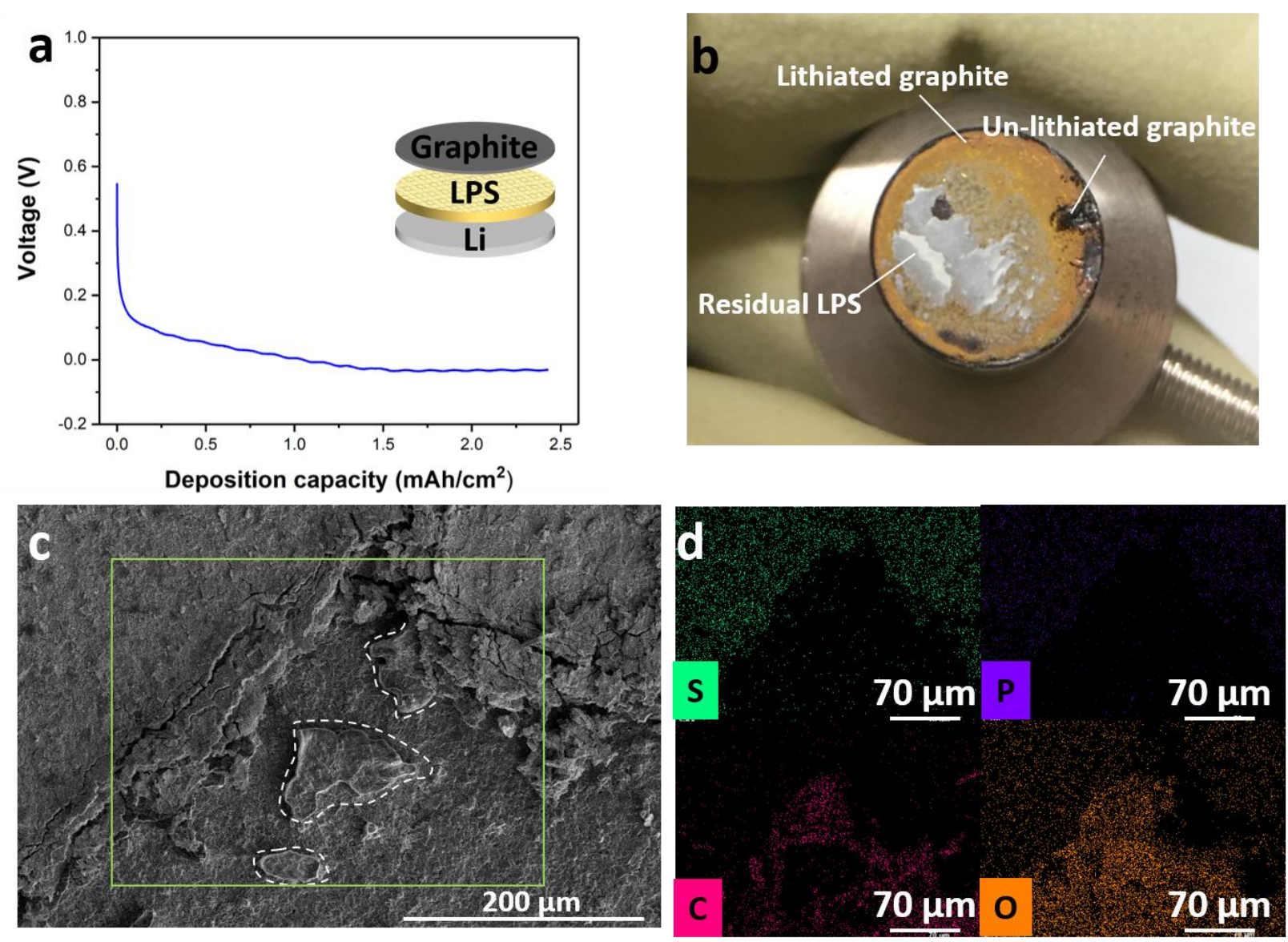

Figure S1. Lithium deposition distribution in pure graphite anode. (a) voltage profile of lithium plating on graphite anode in a Li-graphite cell. (b) image of the surface of dissembled graphite anode after lithium plating. (c) SEM image and (d) EDX mapping of an area from the dissembled anode. 


\section{Discussion Two:}

Considering the 3D anode composite structure, we estimated the maximum lithium metal capacity based on a complete filling of the pores/void in the anode.

The porosity was first calculated. From the SEM image in Figure S1, the thickness of the composite is about $30 \mu \mathrm{m}$. With the density values of graphite $\left(\sim 2.1 \mathrm{~g} \mathrm{~cm}^{-3}\right)$ and LPS $\left(\sim 1.8 \mathrm{~g} \mathrm{~cm}^{-3}\right)$ and mass loadings of graphite $\left(1.7 \mathrm{mg} \mathrm{cm}^{-2}\right)$ and LPS $\left(1.7 \mathrm{mg} \mathrm{cm}^{-2}\right)$, the porosity of the anode is calculated as: porosity $=\frac{30 \mu \mathrm{m}-\left(\frac{1.7 \mathrm{mg} / \mathrm{cm} 3}{2.1 \mathrm{~g} / \mathrm{cm} 3}+\frac{1.7 \mathrm{mg} / \mathrm{cm} 3}{1.8 \mathrm{~g} / \mathrm{cm} 3}\right)}{30 \mu \mathrm{m}}=\sim 41 \%$. The maximum lithium metal capacity will be $30 \mu \mathrm{m} * 41 \% * 1 \mathrm{~cm}^{2} * 2061 \mathrm{mAh} \mathrm{cm}^{-3}=2.53 \mathrm{mAh} \mathrm{cm}^{-2}$. The total anode capacity will be

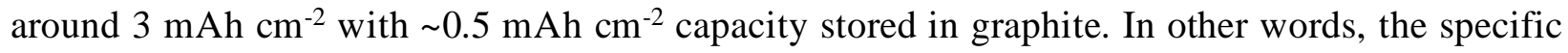
capacity normalized to the graphite weight will be around $1800 \mathrm{mAh} \mathrm{g} \mathrm{g}^{-1}$ and around $900 \mathrm{mAh} \mathrm{g}^{-1}$ based on the combined weight of graphite and LPS.

\section{Discussion Three:}

We also disassembled the 3D anode cell after $0.5 \mathrm{mAh} \mathrm{cm}^{-2}$ of lithium metal deposition after graphite lithiation. The SEM images of a tilted cross-section along with Energy-dispersive X-ray spectroscopy (EDX) mapping are shown in Figure S2. The cross section is between the two marked dashed lines, and the current collector side of the 3D anode is above the upper dashed line. In the EDX mapping images shown in Figure S2b and S2c, the P and S signals represent the LPS SSE, and the carbon signal represents the lithiated graphite. The uniform distribution of lithiated graphite and SSE in the anode network are clearly observed. Similar to the analysis of Figure S1, the oxygen signal represents the summation of lithiated graphite and lithium metal due to brief exposure to air during sample transfer. We attribute the region with strong oxygen signals but weak carbon signals to metallic lithium. 

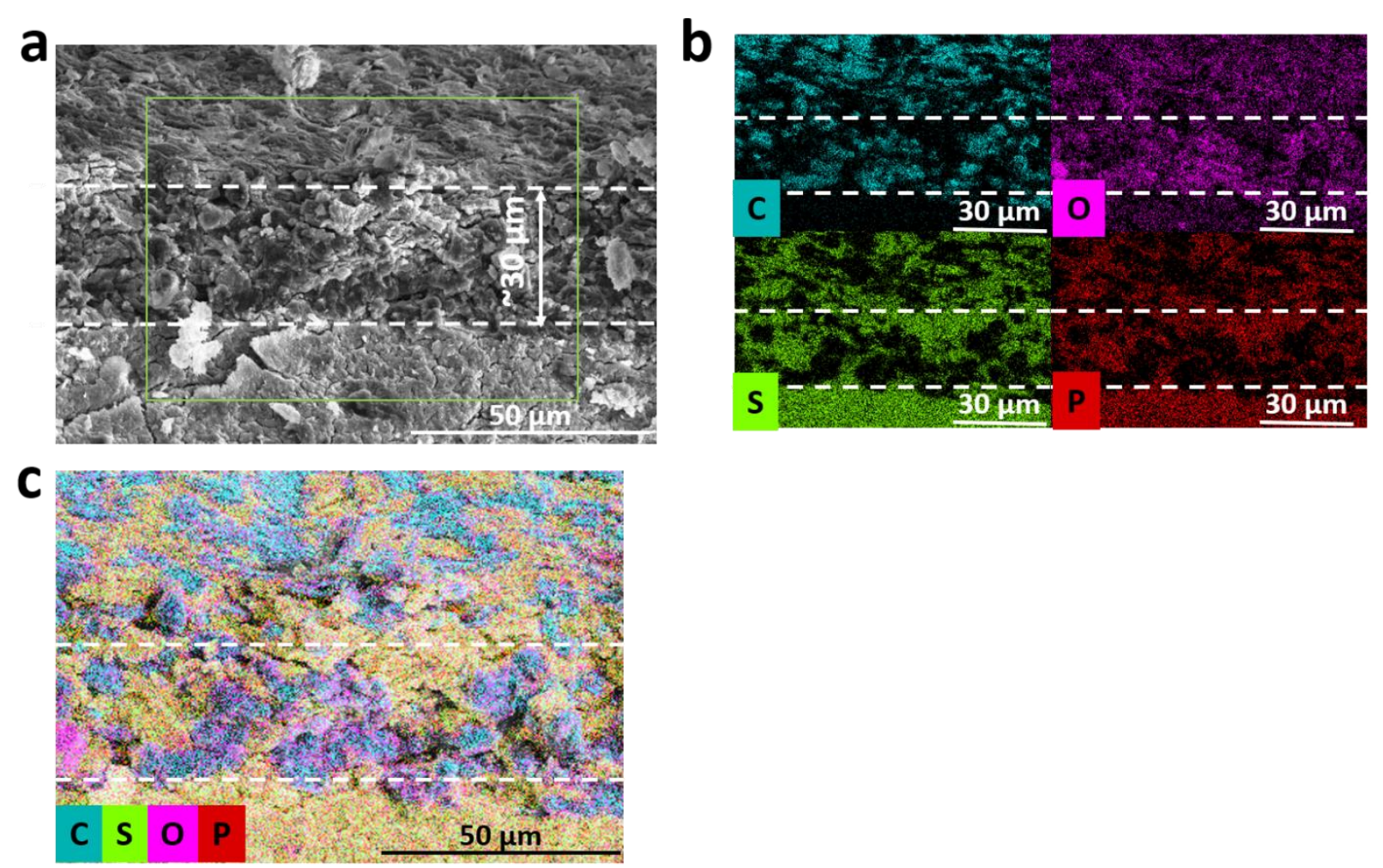

Figure S2. Lithium metal deposition in 3D anode. (a)SEM image and (b), (c) EDX mappings of tilted cross-section of Li-3D anode cell after $1 \mathrm{mAh} \mathrm{cm}^{-2}$ lithium deposition. 
Table S1. Critical current tests summary of all-solid-state batteries

\begin{tabular}{llll}
\hline Cell configuration & $\begin{array}{l}\text { Short-circuit } \\
\text { current density } \\
\text { / } \mathrm{mA} \mathrm{cm}^{-2}\end{array}$ & $\begin{array}{l}\text { Short-circuit } \\
\text { capacity } \\
\text { / mAh cm }\end{array}$ & reference \\
\hline Li-LPS-Li & 0.7 & 0.1 & 8 \\
Li-LiFSI@LPS-Li & 2 & 0.1 & 8 \\
Li-LPS-SS & 0.3 & 0.15 & 9 \\
Li-LPS-Li & $0.4-1$ & $0.4-1$ & 10 \\
Li-LPS-Li & $<0.1$ & $<0.1$ & 11 \\
Li-LLZO-Li & 0.15 & 0.3 & 12 \\
Li-LLZO-Li & 0.9 & 0.45 & 13 \\
Li-LLZO-Li & 0.15 & 0.075 & 14 \\
Li-LLZO-Li & 0.125 & 0.125 & 15 \\
Li-LLZT-Li & 0.15 & 0.15 & 16 \\
Li-LLZT-Li & 0.5 & 0.5 & This work \\
Li-LPS-Li & 0.4 & 0.4 & 17 \\
Li-3D-LPS-3D-Li & 1.4 & 1.4 & \\
\hline & & & 17 \\
\hline
\end{tabular}
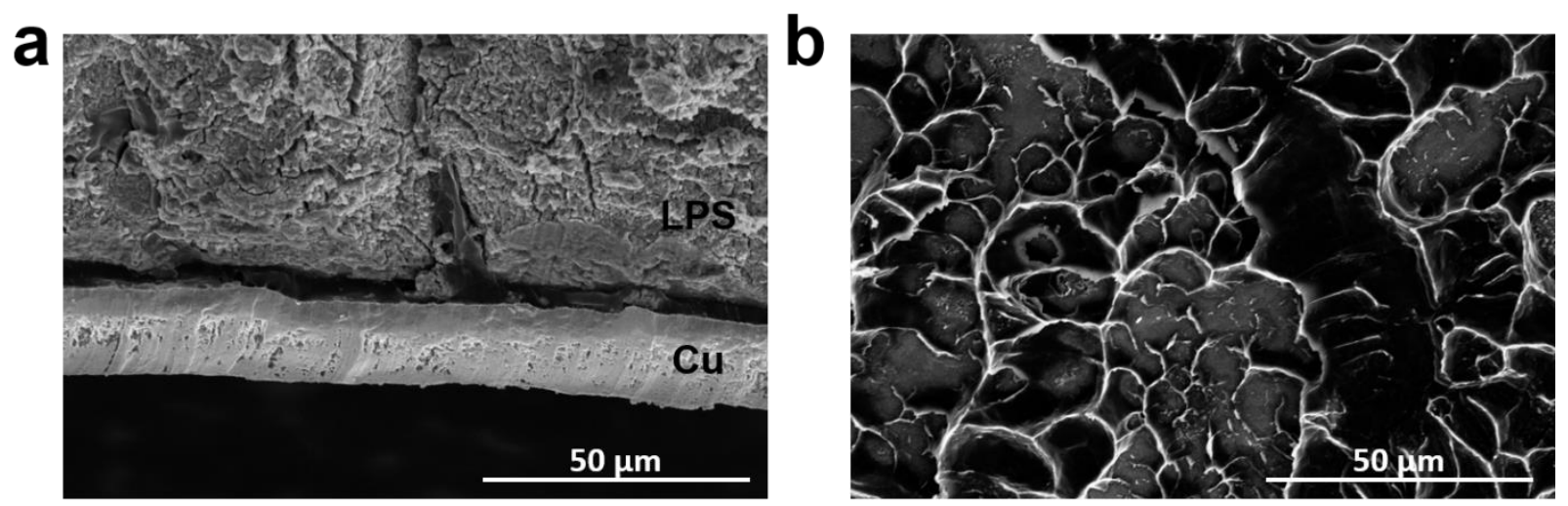

Figure S3. Lithium metal deposition morphology in Li-Cu cell. SEM images of (a) cross section and (b) top view of lithium metal deposition on the $\mathrm{Cu}$ current collector with LPS SE. 

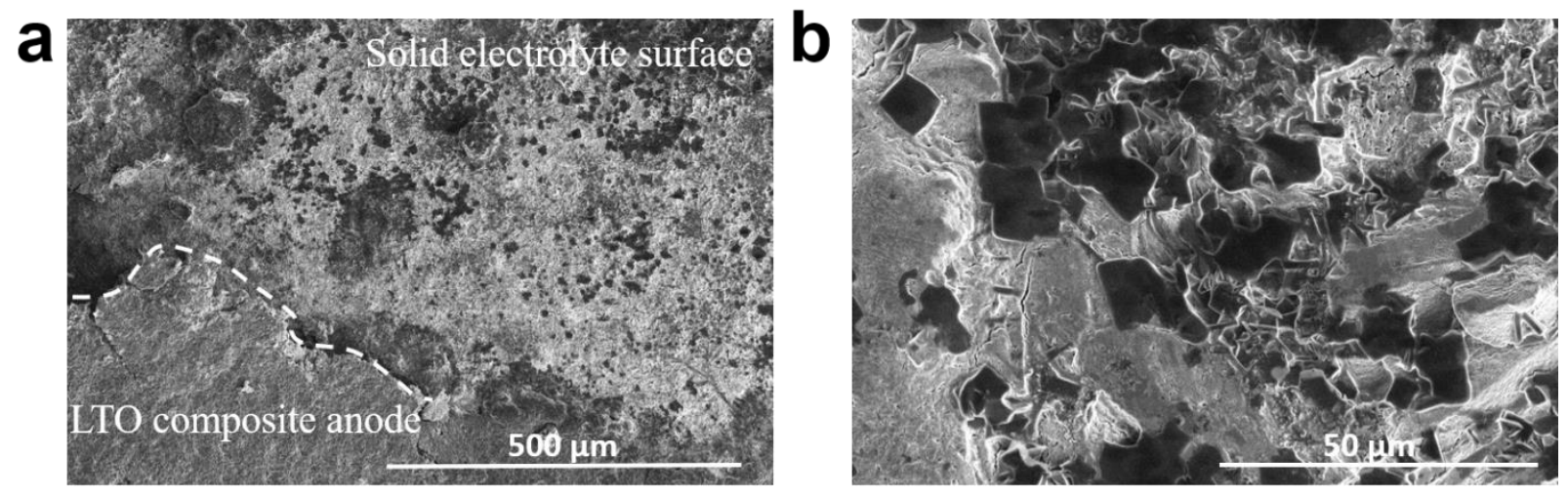

Figure S4. Lithium metal deposition in LTO-based 3D anode. (a) large scale and (b) small scale SEM images of the interface between anode and SE after over-lithiation in LTO-based anode composite cell. The scale bar for the left image is $500 \mu \mathrm{m}$, and $50 \mu \mathrm{m}$ for the right image.
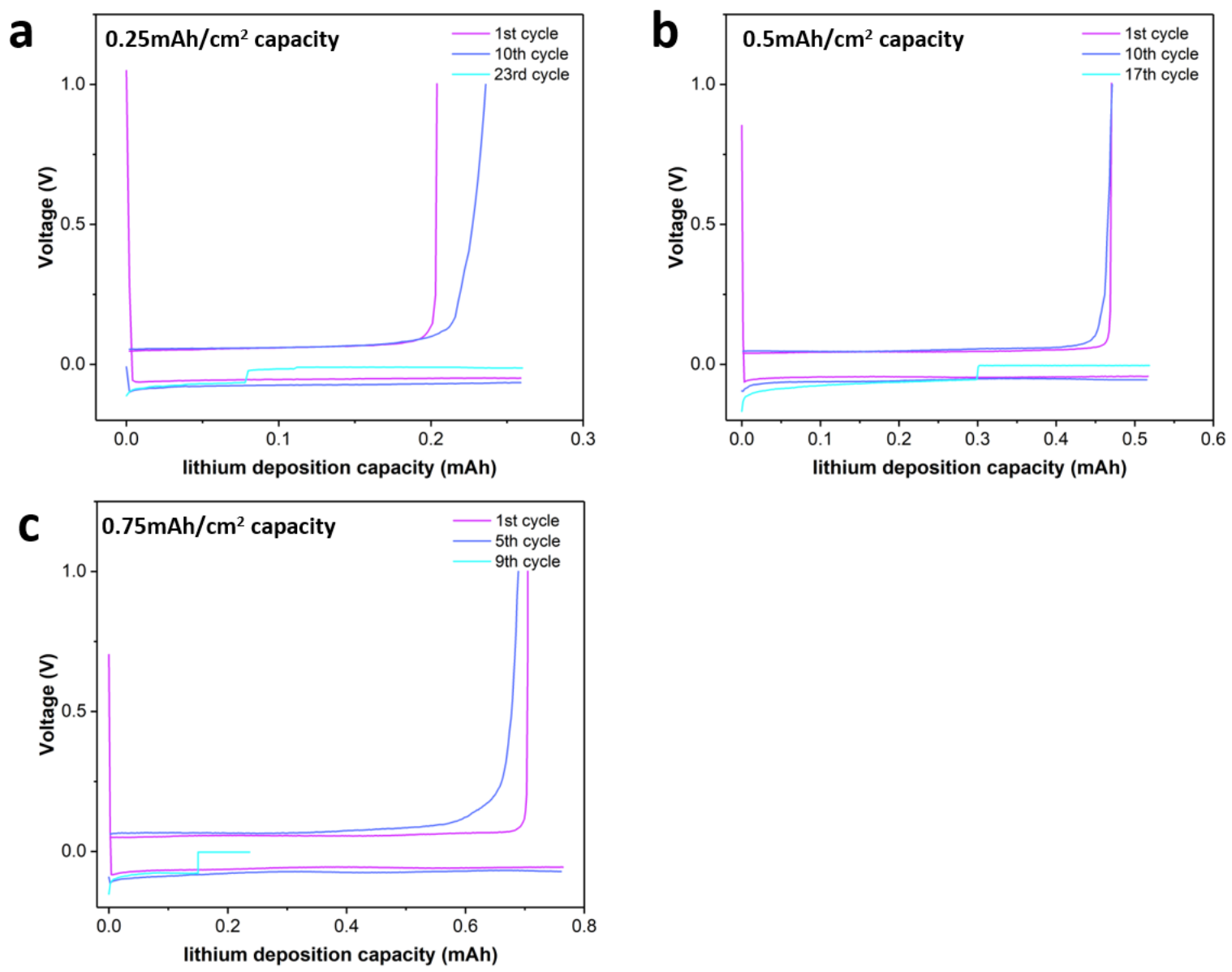

Figure S5. Voltage profiles of Li-Cu cells shown in Figure 3a with (a) $0.25 \mathrm{mAh} \mathrm{cm}^{-2}$, (b) $0.5 \mathrm{mAh}$ $\mathrm{cm}^{-2}$, c) $0.75 \mathrm{mAh} \mathrm{cm}^{-2}$ areal Li plating capacity. 


\section{Discussion Four:}

LPS solid electrolyte was chosen for its high room temperature ionic conductivity $\left(2.01 * 10^{-3} \mathrm{~S}\right.$ $\mathrm{cm}^{-1}$ ), and ease of densification to form composite electrodes and electrolyte layers at low temperatures (low Young's: $\sim 20 \mathrm{GPa}$, shear elastic moduli: $\sim 7 \mathrm{GPa}$. These are the same reasons why LPS-based solid state battery remains a research focus despite the known limitations of its stability issue with Li. Additionally, we have chosen LPS as our electrolyte layer. Using the same electrolyte material for the anode would help minimize any possible chemical reactions between the composite anode and the electrolyte if a different electrolyte for the anode was chosen. Since the main purpose for this study is to demonstrate the graphite-based $3 \mathrm{D}$ anode can enhance the battery cycle life, we were trying to minimize the materials selection in order to focus on the function of graphite.

Post-mortem analysis was performed on the Figure 3 cell to understand its degradation mechanism. The cross-sectional SEM image of the lithiated anode after 20 cycles is shown in Figure S3a. Based on the EDX mapping images shown in Figure S3b and S3c, we concluded that the lithium and graphite distribution remained uniform as a $3 \mathrm{D}$ composite. However, the electrochemical impedance spectroscopy (EIS) results shown in Figure S3 provided a plausible explanation for the cell degradation. In Figure S4a, a Li/LPS/Li symmetric cell was tested first to understand the Li/LPS interface behavior after cycling. The semi-circles shown in Figure S4a correspond to the bulk electrolyte impedance and the interface impedance between LPS and lithium. After 20 cycles, as the fitted values shown in Figure S4a, the Rbulk increased from $1000 \mathrm{Ohm}$ to $1641 \mathrm{Ohm}$ and the Rinterface increased from $166.9 \mathrm{Ohm}$ to $525.4 \mathrm{Ohm}$, indicative of the instability between LPS and lithium. Results for the Li/3D cell are shown in Figure S4b. Again, the semi-circle corresponds to bulk electrolyte impedance and the interface impedance between LPS and both electrodes, while 
the low frequency feature is attributed to the $\mathrm{Li}^{+}$diffusion process in the 3D anode ${ }^{18,19}$. After 20 cycles, the Rbulk increased from $1079 \mathrm{Ohm}$ to $2156 \mathrm{Ohm}$, and Rinterface increased from $29.4 \mathrm{Ohm}$ to 465.2 $\mathrm{Ohm}$. The diffusion tail is also bended down, implying the $\mathrm{Li}^{+}$diffusion in $3 \mathrm{D}$ anode appeared to have deteriorated after cycling. As reported previously, the instability of LPS vs Li metal is the likely reason for both electrolyte bulk impedance and the interface impedance increase and slower diffusion ${ }^{18-23}$.
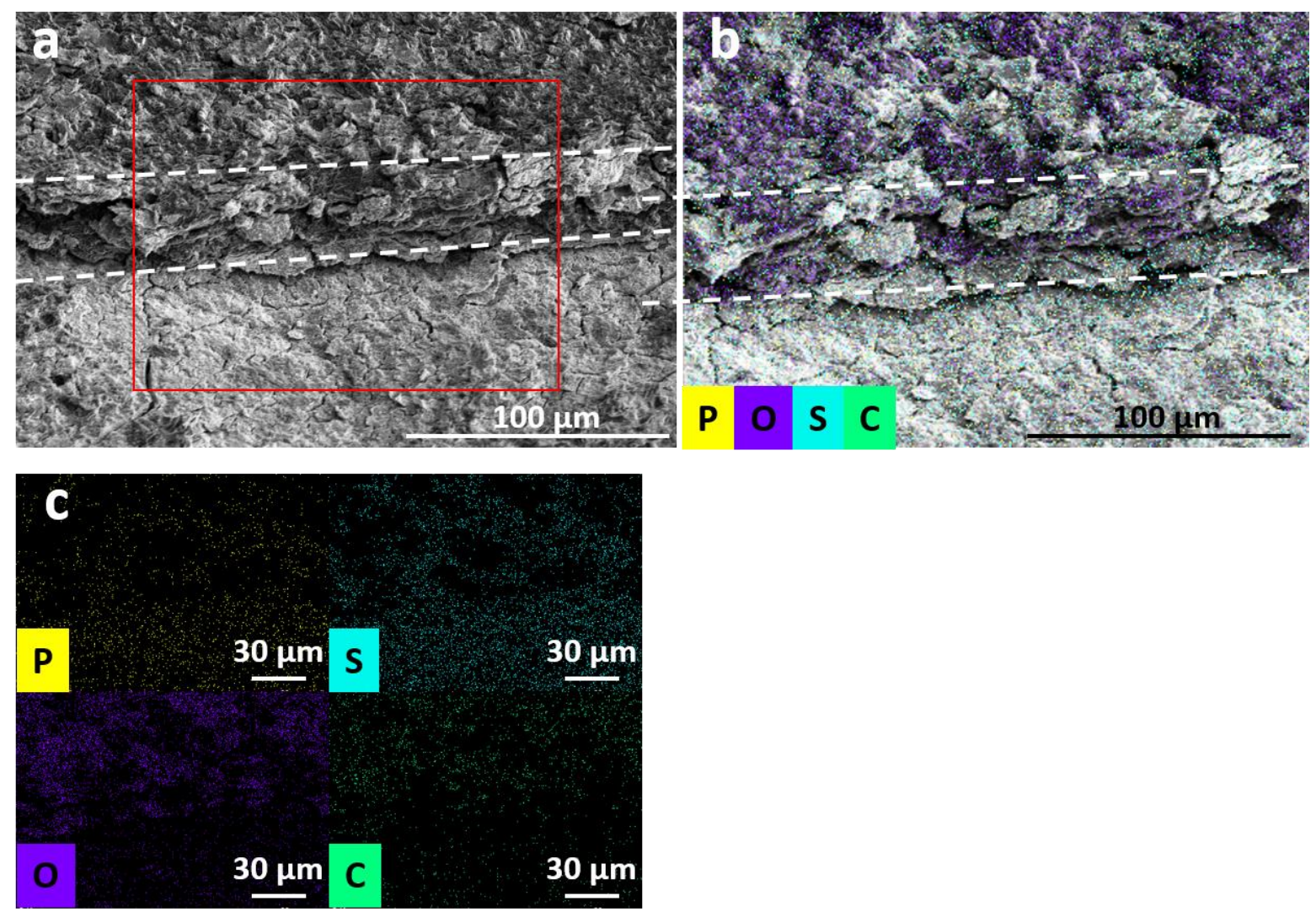

Figure S6. Cycled 3D anode structure. (a) SEM image and (b), (c) EDX mappings of tilted cross section of hybrid anode after 20 cycles, which is the same cell as shown in Figure 4. 

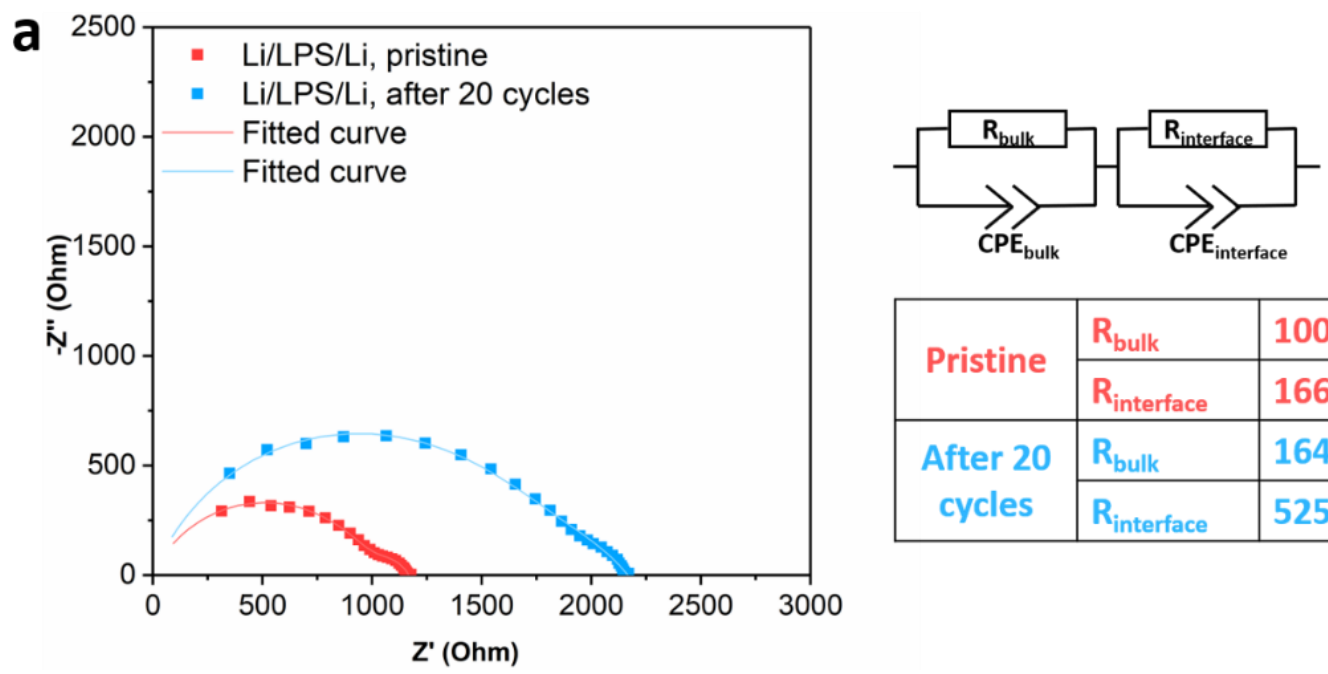

\begin{tabular}{|c|l|l|}
\hline \multirow{2}{*}{ Pristine } & $R_{\text {bulk }}$ & 1000 Ohm \\
\cline { 2 - 3 } & $R_{\text {interface }}$ & $166.9 \mathrm{Ohm}$ \\
\hline \multirow{2}{*}{$\begin{array}{c}\text { After 20 } \\
\text { cycles }\end{array}$} & $\mathrm{R}_{\text {bulk }}$ & $1641 \mathrm{Ohm}$ \\
\cline { 2 - 3 } & $\mathrm{R}_{\text {interface }}$ & $525.4 \mathrm{Ohm}$ \\
\hline
\end{tabular}
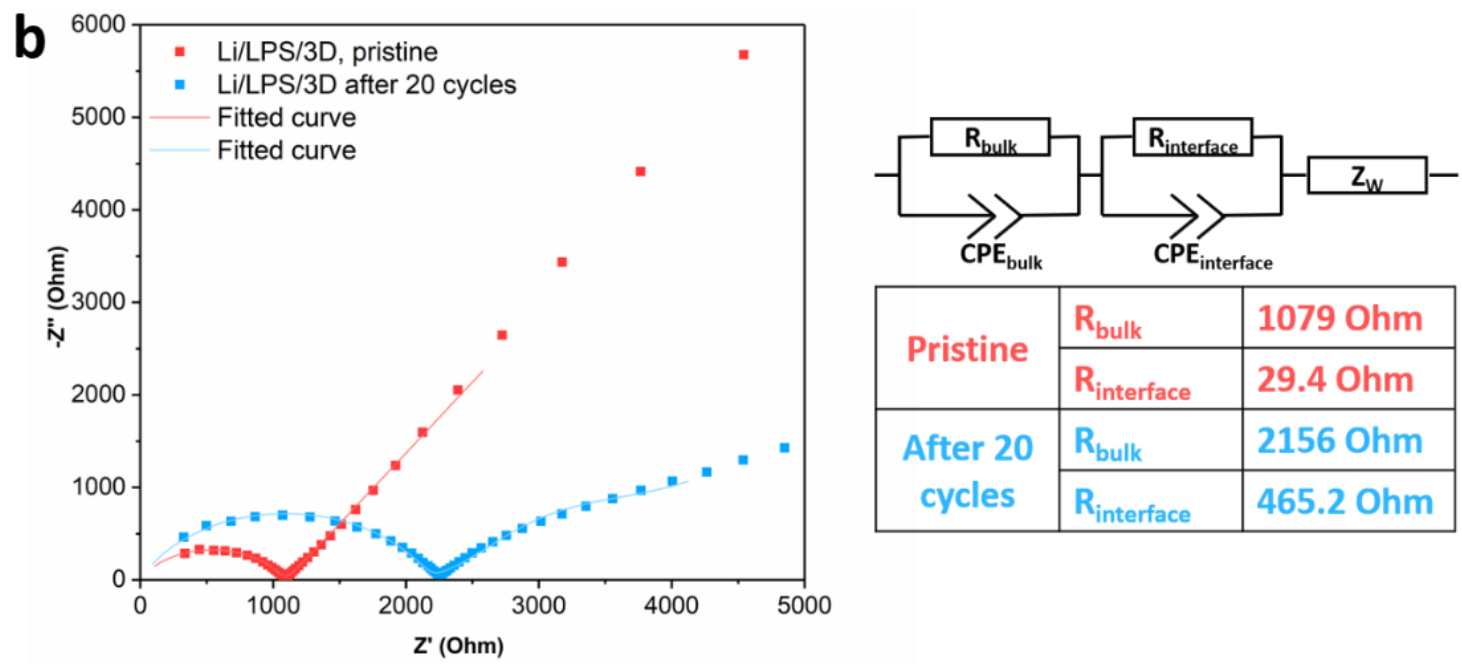

Figure S7. Impedance plots with fitting results of (a) a Li-Li symmetric cell and (b) a Li-3D anode cell before and after 20 cycles. The fitted values of $\mathrm{R}_{\text {bulk }}$ and $\mathrm{R}_{\text {interface }}$ are listed in the table. The measurements were performed at $25^{\circ} \mathrm{C}$ in a frequency range from $7 \mathrm{MHz}$ to $10 \mathrm{mHz}$.

Discussion Five:

We calculated the respective coulombic efficiency for the graphite portion and lithium metal portion in the 3D anode in a 3D-NCA cell. Using the data shown in the Figure 4 with G/N=4:3, we could calculate the graphite-NCA cycling $\mathrm{CE}$ in this Li-ion cell with the equation: $\mathrm{CE}=$ (remaining cyclable lithium capacity / initial cyclable lithium capacity) ${ }^{-c y c l e ~ n u m b e r}$, where the discharge capacity is used as a measure for cyclable lithium capacity. From the 2 nd cycle to the 
50th cycle, the average graphite-NCA CE is about 99.07\%. For the cell in Figure 4 with G/N=2:3, there is $\sim 0.5 \mathrm{mAh} \mathrm{cm}^{-2}$ capacity stored in graphite and $\sim 0.25 \mathrm{mAh} \mathrm{cm}^{-2}$ capacity stored in lithium metal. The cell retained $38 \%$ capacity after 80 cycles. Assuming the graphite part of the 3D anode maintained the same CE of $99.07 \%$, there was $0.5 *(99.07 \%)^{79}=0.239 \mathrm{mAh} \mathrm{cm}^{-2}$ capacity of active lithium left in graphite after 80 cycles. Thus, there was $0.75 * 0.38-0.239=0.046 \mathrm{mAh} \mathrm{cm}^{-}$ ${ }^{2}$ stored as lithium metal after 80 cycles. The lithium metal cycling CE is then $(0.046 / 0.25)^{-79}=$ $97.87 \%$.

Discussion Six:

We recognize that $\mathrm{Cu}$ corrosion could occur when the anode is completely free of lithium (either as metal or intercalated into graphite). As a result, we tried using a Ti current collector instead of $\mathrm{Cu}$ with the same cell configuration as the NCA-Cu cell, considering Ti shows a better stability vs LPS.$^{7}$ The performance is shown in Figure R 1 below. The cell lost $42 \%$ capacity after 15 cycles with a $92 \%$ average $\mathrm{CE}$, which is similar as the performance of the NCA-Cu cell. In this case, we believe the interfacial contact between LPS and Li metal anode is responsible for the degradation rather than $\mathrm{Cu}$ current collector by itself. This is understandable since when $\mathrm{Cu}$ is deposited with $\mathrm{Li}$, it is always anodically protected. We agree, however, if a $\mathrm{Li}$-metal free cell is fabricated and left at open circuit for extended period of time, corrosion of $\mathrm{Cu}$ will likely take place. In that case, the use of $\mathrm{Ti}$ or stainless steel will be more appropriate. 


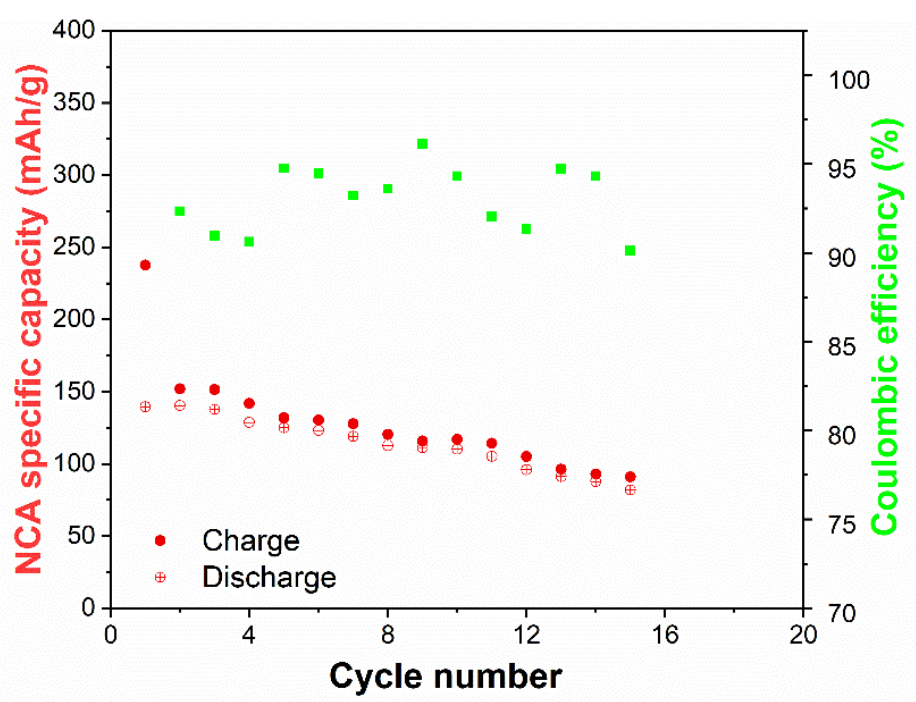

Figure S8. Electrochemical performance of an NCA-Ti cell.
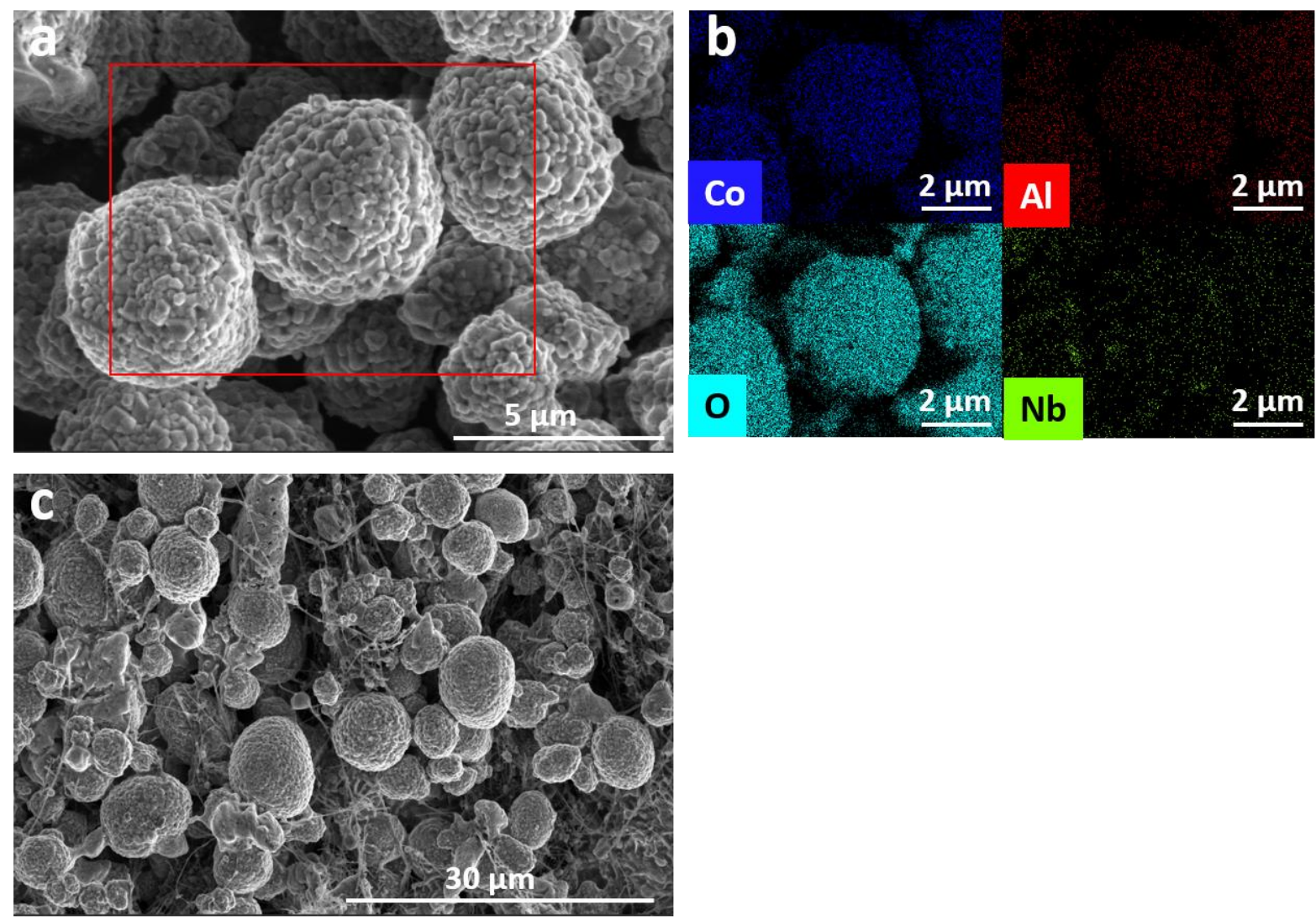

Figure S9. NCA cathode material morphology. (a) SEM image and (b) EDX mapping of coated NCA cathode material, (c) SEM image of NCA cathode composite. 

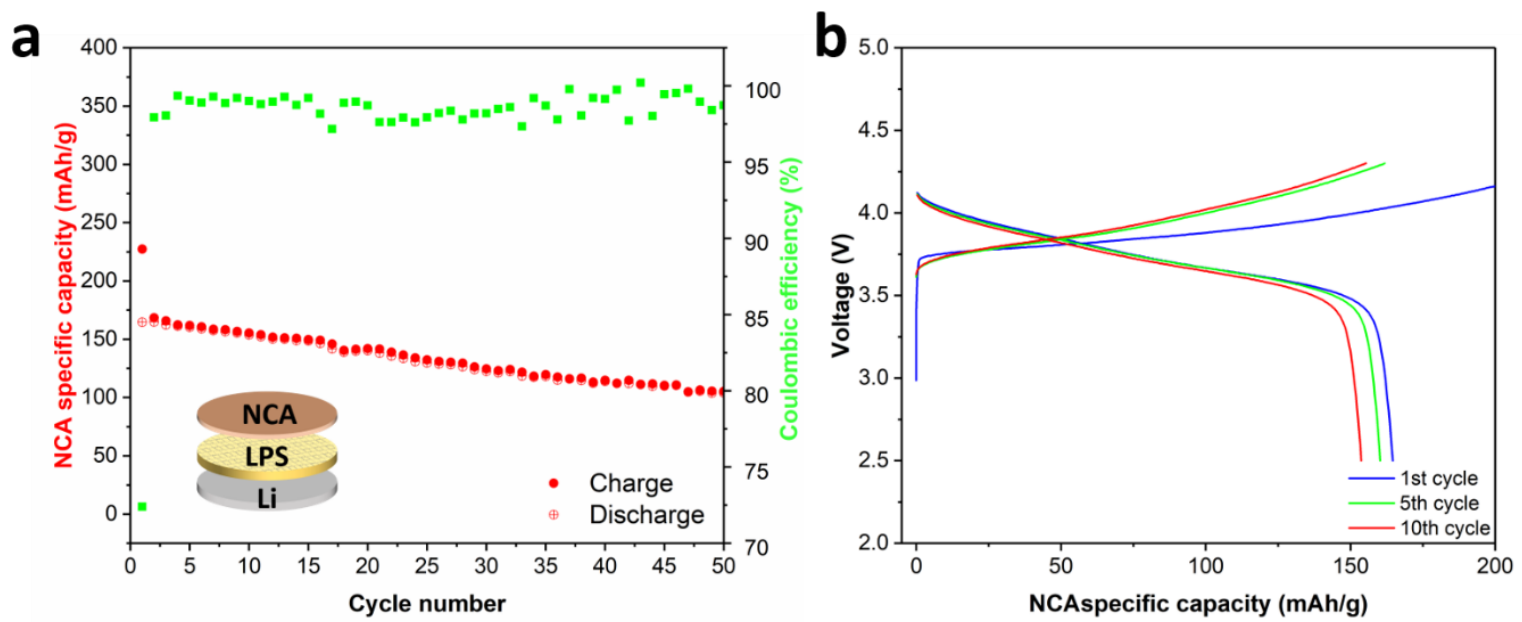

Figure S10. Electrochemical behavior of NCA cathode. (a) Cycling performance and (b) voltage profiles of NCA-Li cell with LPS SSE. The cycling current density is $0.1 \mathrm{mAcm}^{-2}$ in a range of $2.5-4.3 \mathrm{~V}$. 

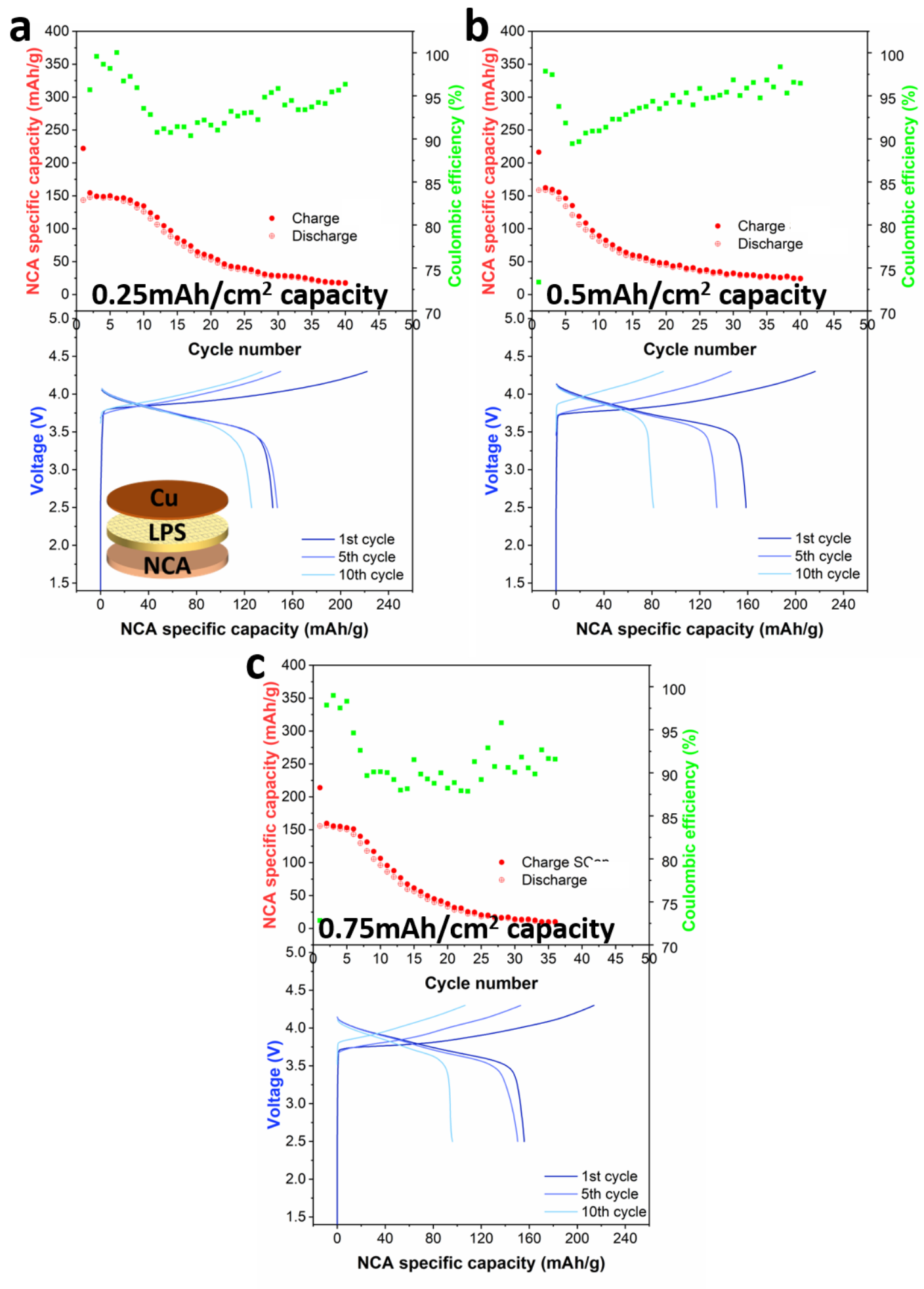

Figure S11. Electrochemical performance of NCA-Cu cells. Cycling performance and voltage profiles of the NCA-Cu cell with (a) $0.25 \mathrm{mAh} \mathrm{cm}^{-2}$; (b) $0.5 \mathrm{mAh} \mathrm{cm}^{-2}$; and (c) $0.75 \mathrm{mAh} \mathrm{cm}^{-2}$ areal deposition capacity. 

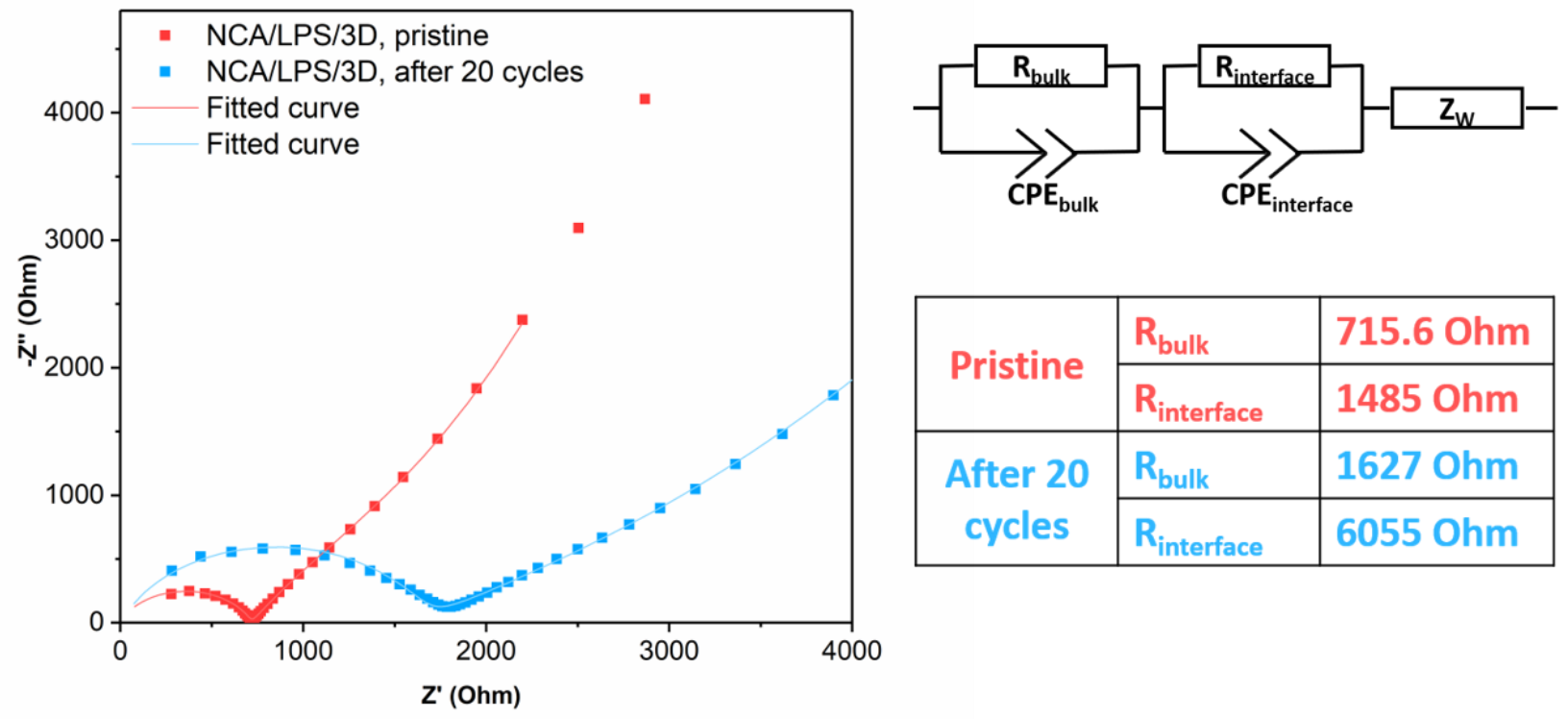

\begin{tabular}{|c|l|l|}
\hline \multirow{2}{*}{ Pristine } & $\mathrm{R}_{\text {bulk }}$ & 715.6 Ohm \\
\cline { 2 - 3 } & $\mathrm{R}_{\text {interface }}$ & 1485 Ohm \\
\hline \multirow{2}{*}{$\begin{array}{c}\text { After 20 } \\
\text { cycles }\end{array}$} & $\mathrm{R}_{\text {bulk }}$ & 1627 Ohm \\
\cline { 2 - 3 } & $\mathrm{R}_{\text {interface }}$ & 6055 Ohm \\
\hline
\end{tabular}

Figure S12. Impedance plots with fitting results of an NCA-3D anode cell before and after 20 cycles, which is the same cell as the one shown in Figure 4 . The fitted values of $\mathrm{R}_{\text {bulk }}$ and $\mathrm{R}_{\text {interface }}$ are listed in the table. The measurements were performed at $25{ }^{\circ} \mathrm{C}$ in a frequency range from 7 $\mathrm{MHz}$ to $10 \mathrm{mHz}$. 
Table S2. Energy density projection of NCA-3D anode cells with different G/N ratios*

\begin{tabular}{|c|c|c|c|c|c|}
\hline $\mathrm{G} / \mathrm{N}$ ratio & $\begin{array}{l}\text { Weight of } \\
\text { anode } \\
/ \mathrm{mg}\end{array}$ & $\begin{array}{l}\text { Weight of } \\
\mathrm{SE} \\
/ \mathrm{mg}\end{array}$ & $\begin{array}{l}\text { Weight of } \\
\text { cathode } \\
/ \mathrm{mg}\end{array}$ & $\begin{array}{l}\text { Average } \\
\text { voltage } \\
\text { /V }\end{array}$ & $\begin{array}{l}\text { Energy } \\
\text { density } \\
/ \mathrm{Wh} \mathrm{g}^{-1}\end{array}$ \\
\hline 3 & 57.27 & 20 & 20 & 3.5 & 75.6 \\
\hline 2 & 39.18 & 20 & 20 & 3.5 & 83.8 \\
\hline $3 / 2$ & 28.63 & 20 & 20 & 3.5 & 94.0 \\
\hline 1 & 19.09 & 20 & 20 & 3.5 & 107.1 \\
\hline $2 / 3$ & 12.73 & 20 & 20 & 3.55 & 141.4 \\
\hline $2 / 4$ & 9.54 & 20 & 20 & 3.62 & 153.4 \\
\hline $2 / 5$ & 7.63 & 10 & 20 & 3.68 & 205.4 \\
\hline $2 / 5$ & 7.63 & 20 & 20 & 3.68 & 162.2 \\
\hline $2 / 5$ & 7.63 & 50 & 20 & 3.68 & 99.5 \\
\hline $2 / 5$ & 7.63 & 100 & 20 & 3.68 & 60.6 \\
\hline $2 / 5$ & 7.63 & 200 & 20 & 3.68 & 33.9 \\
\hline Anode free & 0 & 20 & 20 & 3.75 & 196.8 \\
\hline
\end{tabular}

*NCA-3D cell information: SE: weight :20 mg, thickness: $\sim 100 \mu \mathrm{m}$.

Graphite anode: specific capacity $220 \mathrm{mAh} \mathrm{g}^{-1}$

NCA Cathode: specific capacity $150 \mathrm{mAh} \mathrm{g}^{-1}$

Anode composition: LPS 50wt $\%$, graphite 50wt $\%$

Cathode composite composition: NCA 70wt\%, LPS $28 \mathrm{wt} \%$ and VGCF ((vapor growth carbon fiber).) $2 \mathrm{wt} \%$ 